\title{
SPDT GaAs Switches With Copper Metallized Interconnects
}

\author{
Y. C. Wu, E. Y. Chang, Senior Member, IEEE, Y. C. Lin, H. T. Hsu, S. H. Chen, W. C. Wu, L. H. Chu, and \\ C. Y. Chang, Fellow, IEEE
}

\begin{abstract}
Copper metallized AlGaAs/InGaAs psedomophic high-electron-mobility transistor (PHEMT) single-pole-doublethrow (SPDT) switches utilizing platinum (Pt, $70 \mathrm{~nm})$ as the diffusion barrier is reported for the first time. In comparison with the Au metallized switches, the Cu metallized SPDT switches exhibited comparable performance with insertion loss of less than $0.5 \mathrm{~dB}$, isolation larger than $35 \mathrm{~dB}$ and the input power for one dB compression (input $P_{1 \mathrm{~dB}}$ ) of $27 \mathrm{dBm}$ at $2.5 \mathrm{GHz}$. These switches were annealed at $250^{\circ}$ for $20 \mathrm{~h}$ for thermal stability test and showed no degradation of the dc characteristics after the annealing. Also, after $144 \mathrm{~h}$ of high temperature storage life (HTSL) environment test, these switches still remained excellent and reliable radio frequency (RF) characteristics. It is successfully demonstrated for the first time that the copper metallization using $\mathrm{Pt}$ as the diffusion barrier could be applied to the GaAs monolithic microwave integrated circuits switch fabrication with good RF performance and reliability.
\end{abstract}

Index Terms-Copper metallization, GaAs psedomophic high-electron-mobility transistor (PHEMT), platinum, singlepole-double-throw (SPDT), switch.

\section{INTRODUCTION}

$\mathbf{T}$ HE COPPER metallization process has been widely used in silicon integrated circuit technology since IBM announced its success with copper metallization in the silicon very large scale integration process [1]-[3]. Traditionally, $\mathrm{Ti} / \mathrm{Au}$ interconnect metal is widely used for the fabrication of GaAs based field effect transistors and monolithic microwave integrated circuits (MMICs) and the reliability of the metal system has been well proven. In this study, we use copper metal instead of gold metal as the interconnect metal for the AlGaAs/InGaAs psedomophic high-electron-mobility transistor (PHEMT) single-pole-double-throw (SPDT) switches. The employment of copper as the metallization metal has several major advantages over gold, such as lower resistivity, higher thermal conductivity, and lower cost. Assuming that copper replaces gold as the metallization for a switch, significant improvement in the electrical conductivity can increase the switch speed of the circuit.

Manuscript received June 5, 2006; revised October 23, 2006. This work was supported by the National Science Council of Taiwan, R.O.C., and the Ministry of Economic Affairs under Contracts NSC 94-2752-E-009-001-PAE and 94-EC-17-A-05-S1-020.

Y. C. Wu, E. Y. Chang, Y. C. Lin, S. H. Chen, W. C. Wu, and L. H. Chu are with the Department of Materials Science and Engineering, National Chiao Tung University, Hsinchu 30010, Taiwan, R.O.C. (e-mail: edc@ @ail.nctu.edu. tw).

H. T. Hsu is with the Department of Communications Engineering, Yuan Ze University, Chungli Taoyuan 320, Taiwan, R.O.C.

C. Y. Chang is with the Department of Electronics Engineering, National Chiao Tung University, Hsinchu 30010, Taiwan, R.O.C.

Digital Object Identifier 10.1109/LMWC.2006.890340
Nevertheless, copper diffuses very fast into GaAs if without any diffusion barrier and forms a deep acceptor to capture carrier in GaAs. It will lead to the failure of the electrical properties of the GaAs devices. In our previous research, we have demonstrated backside copper metallization on GaAs semiconductor field-effect transistors (MESFET) using TaN as the diffusion barrier [4], copper airbridge on low noise GaAs high-electron-mobility transistors (HEMTs) using $\mathrm{WNx}$ as the diffusion barrier [5], and fully copper metallized $\mathrm{InGaP} / \mathrm{GaAs}$ heterojunction bipolar transistor (HBT) using platinum as the diffusion barrier [6]. In this study, we choose Pt as the diffusion barrier metal since $\mathrm{Pt}$ is commonly used as the plate metal for the metal-insulator-metal (MIM) capacitor of the switch. The Pt diffusion barrier was very effective in preventing copper from diffusing into the conventional Schottky and Ohmic metal in this study. The fabrication and the electrical performance of the SPDT MMIC switches using copper metallization technology are reported for the first time.

\section{Device Structure And Fabrication}

The PHEMT wafer was grown by metal organic chemical vapor deposition (MOCVD) on a 4-in semi-insulating GaAs substrate. The epi-layers of the device, from bottom to top, are composed of a 600-nm buffer, a 13-nm InGaAs channel, a 3-nm undoped AlGaAs spacer, a delta-doped layer, and a 37-nm AlGaAs schottky layer and 60-nm n-GaAs capping layer. The Hall electron mobility and sheet carrier concentrations at room temperature are $6500 \mathrm{~cm}^{2} / \mathrm{V} \cdot \mathrm{s}$ and $3.0 \times 10^{12} \mathrm{~cm}^{-2}$, respectively.

The device fabrication was carried out by the use of traditional lithograthy and lift-off technique. Mesa isolation and epitaxial resistors were formed utilizing $\mathrm{H}_{3} \mathrm{PO}_{4}-\mathrm{H}_{2} \mathrm{O}_{2}-\mathrm{H}_{2} \mathrm{O}$ mixing solution. The source and drain Ohmic metals $(\mathrm{Au} / \mathrm{Ge} / \mathrm{Ni} / \mathrm{Au})$ were deposited by e-beam evaporator and rapid thermal annealed at $410{ }^{\circ} \mathrm{C}$ for $30 \mathrm{~s}$ to form Ohmic contacts. The typical Ohmic contact resistance was $0.12 \Omega \cdot \mathrm{mm}$. A mixing solution of citric acid and hydrogen peroxide was used for the gate-recess process. Ti/Pt/Au was used as the gate metal and the gate length of the devices was $0.5 \mu \mathrm{m}$. The gate was formed by electron beam evaporation and lift-off process. The silicon nitride films were used for passivation and as the dielectric for capacitors and were deposited by plasma enhanced chemical vapor deposition (PECVD). The capacitance per unit area of the MIM capacitor was $0.25-\mathrm{fF} / \mu \mathrm{m}^{2}$ and the thickness of the dielectric was $200 \mathrm{~nm}$. The first metal consists of the adhesion layer Ti $(30 \mathrm{~nm})$ and the diffusion barrier Pt $(70 \mathrm{~nm})$, which was also used as the top plate of a MIM capacitor. Finally, a $2-\mu \mathrm{m}$ thick copper metal for interconnection was electroplated and a 100-nm thick silicon nitride was deposited as the passivation layer. 


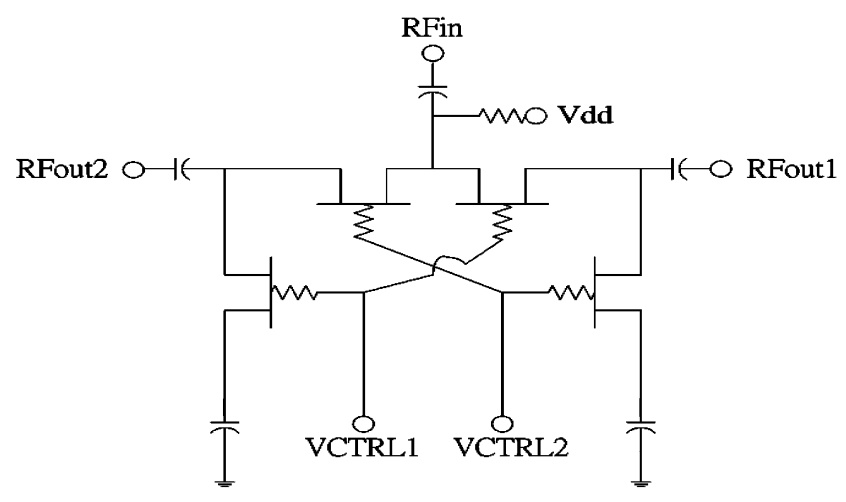

Fig. 1. Circuit schematic of the series/shunt SPDT switch.

The dc and RF electrical characteristics of the copper switches were characterized to demonstrate the practicability of using the copper interconnection on the SPDT switches. Thermal stability of the Pt diffusion barrier is a major concern in this study. To demonstrate the reliability of the copper switches with the Pt diffusion barrier, the devices with the copper interconnects were furnace annealed at $250^{\circ}$ for $20 \mathrm{~h}$ in the nitrogen atmosphere. The dc characteristics before and after thermal annealing were compared to verify the thermal stability of the copper metallized switches. Besides, the long-term thermal stress of copper switches was evaluated by using HTSL method, which was carried out by annealing at $150^{\circ}$ for $144 \mathrm{~h}$ with no bias [7]-[10].

\section{RESUltS AND Discussion}

The switch developed in this study can be used in the wireless LAN system for switching between the transmitting and receiving modes. In this study, the series/shunt SPDT switch uses $0.5-\mu \mathrm{m}$ gate length, dual-fingers AlGaAs/InGaAs PHEMTs. The gate resistor between the signal and control terminal has a value of $3 \mathrm{k} \Omega$. Fig. 1 exhibits the circuit schematic of the series/shunt SPDT switch. When the control voltage 1 ( $\left.V_{\text {CTRL1 }}\right)$ is biased at $3 \mathrm{~V}$ and the control voltage $2\left(V_{\mathrm{CTRL} 2}\right)$ at $0 \mathrm{~V}$, the $R F$ signal is flowing from $R F_{\text {in }}$ to $R F_{\text {out1 }}$. Oppositely, the $R F$ signal path is from $\mathrm{RF}_{\text {in }}$ to $\mathrm{RF}_{\text {out2 }}$ as $V_{\mathrm{CTRL}}$ is biased at $0 \mathrm{~V}$ and $V_{\mathrm{CTRL} 2}$ at $3 \mathrm{~V}$.

Compared with the HEMTs in the switch fabricated with conventional gold interconnects, the HEMTs in the switch fabricated using copper metallization in this study showed similar dc characteristics. The HEMTs used in the copper SPDT switches exhibited a drain saturation current density of $160 \mathrm{~mA} / \mathrm{mm}$ and a transconductance of $140 \mathrm{mS} / \mathrm{mm}$ at $V_{\mathrm{DS}}=3 \mathrm{~V}$. The devices had threshold voltage of $-1.5 \mathrm{~V}$. The performances of insertion loss and isolation of the SPDT switches with copper metallization and with gold metallization were measured at $2.5 \mathrm{GHz}$, respectively, and the results are as shown in Fig. 2. The copper metallized switch had an insertion loss of $0.33 \mathrm{~dB}$ and an isolation of $36.7 \mathrm{~dB}$ (Control Voltage $=+3 / 0 \mathrm{~V}$, Input Power $=$ $0 \mathrm{dBm})$ at $2.5 \mathrm{GHz}$. The results in the RF characteristics showed very little deviation for the switches with copper interconnects and the switches with gold interconnects. The deviation of these two switches is primarily due to the nonuniformity of the wet chemical etch in the gate recess process, which may influence the on-state resistance and off-state capacitance of the transistor

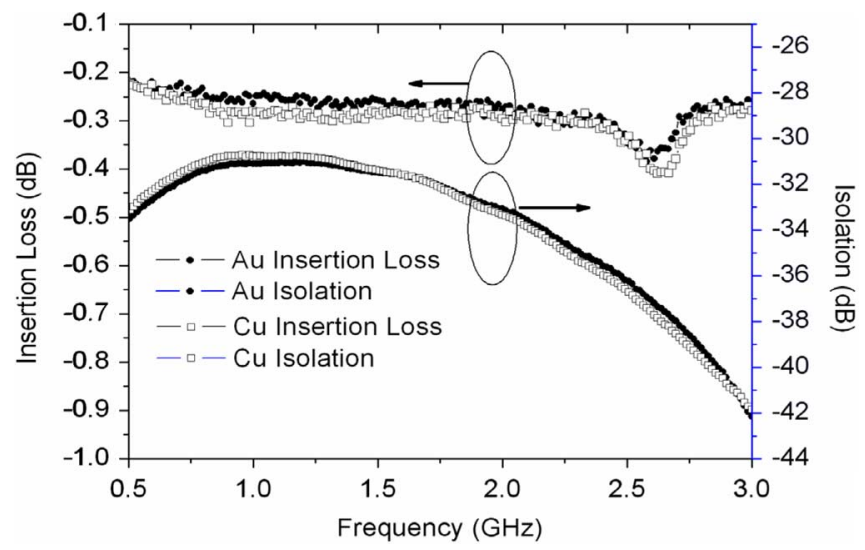

Fig. 2. Insertion loss and isolation vs frequency of the SPDT switches with copper and gold metallizations.

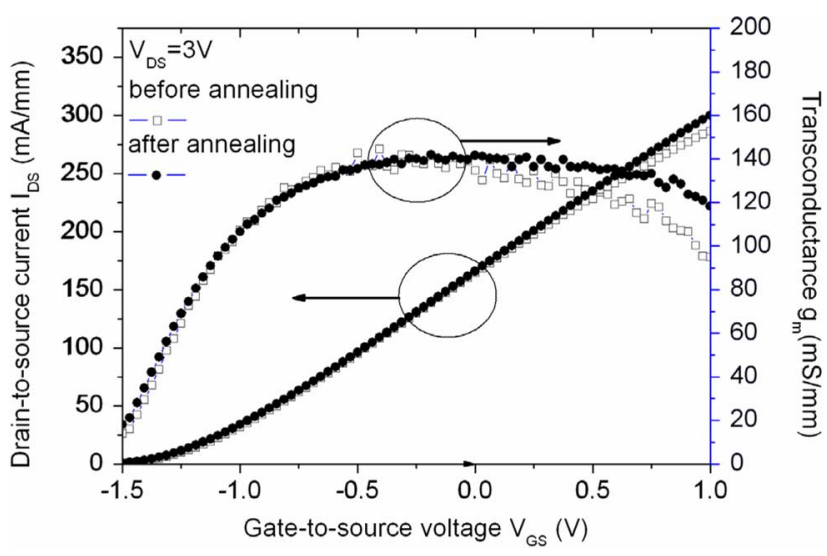

Fig. 3. Transconductance and drain-source current versus $V_{\mathrm{GS}}$ bias characteristics of the copper metallized AlGaAs/InGaAs PHEMT SPDT switches for $0.5-\mu \mathrm{m}$ gate length before and after annealing at $250^{\circ} \mathrm{C}$ for $20 \mathrm{~h}$.

dominating insertion loss and isolation. These RF results were consistent with the dc characteristics, which indicates that the use of copper metallization would not increase the on-state resistance and the off-state capacitance of the active channel of the transistors and implies that the copper metallization could be applied to the interconnects of the SPDT switches without effecting the switch performance.

\section{Reliability Test}

To test the thermal stability of the Pt diffusion barrier, the copper metallized switches were annealed at $250{ }^{\circ} \mathrm{C}$ for $20 \mathrm{~h}$, the dc characteristics of the HEMT device in the circuits after annealing are shown in Fig. 3. The device performance exhibited very little change (less than $5 \%$ difference in transconductance) after thermal annealing. The result demonstrates that the $\mathrm{Cu}-\mathrm{Pt}$ interconnect layers are quite stable and the platinum is an effective diffusion barrier to impede the copper diffusion after thermal annealing. Overall, the copper metallized SPDT switches have sustained the high temperature environment test without obvious de performance degradation.

The RF performance of the copper switches was also evaluated at high temperature. As shown in Fig. 4, the switch has an insertion loss of $0.33 \mathrm{~dB}$ and an isolation of $36.7 \mathrm{~dB}$ at $2.5 \mathrm{GHz}$ before thermal test. After $144 \mathrm{~h}$ of HTSL evaluation at $150{ }^{\circ} \mathrm{C}$ 


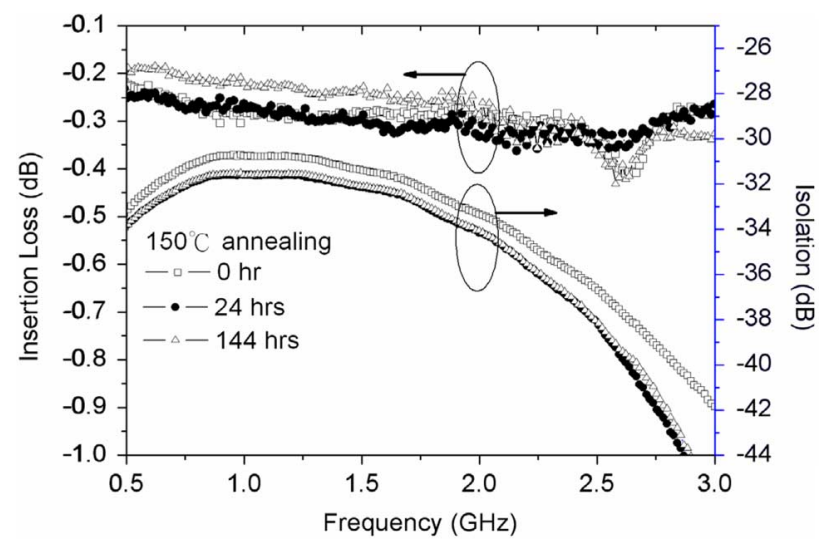

Fig. 4. Insertion loss and isolation of the copper metallized SPDT switches at $2.5 \mathrm{GHz}$ after annealing at $150{ }^{\circ} \mathrm{C}$ for different hours.

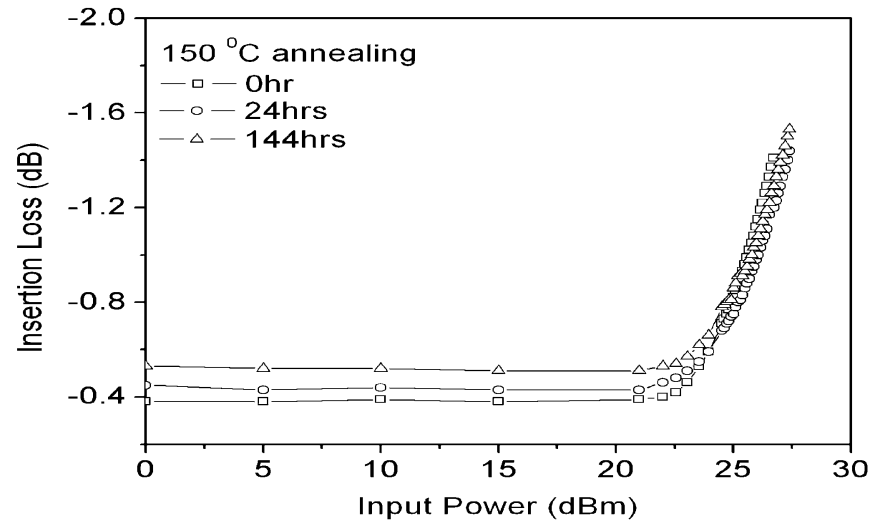

Fig. 5. Insertion loss of the copper metallized SPDT switches with different input power levels at $2.5 \mathrm{GHz}$ after annealing at $150^{\circ} \mathrm{C}$ for different annealing periods.

under nitrogen atmosphere, the copper switches still demonstrated very good reliability with similar RF performance. The Pout-Pin relationships of the copper switches at $2.5 \mathrm{GHz}$ after annealing at $150{ }^{\circ} \mathrm{C}$ for different times are as shown in Fig. 5, input $P_{1 \mathrm{~dB}}$ was kept at about $27 \mathrm{dBm}$ without significant change. Fig. 6 revealed that input third-order intermodulation intercept point (input IP3) of $50 \mathrm{dBm}$ can be achieved at the input power of $15 \mathrm{dBm}$, and the trend of control current still remained stable. It suggests that no copper diffusion into the active device region, and no degradation of on-state resistance and power handing capability for the transistors after thermal annealing occurred for the copper metallized SPDT switches using $\mathrm{Pt}$ as the diffusion barrier.

\section{CONCLUSION}

An SPDT GaAs switch fabricated with copper metallized interconnects using $\mathrm{Pt}$ as the diffusion barrier is reported for the first time. The RF characteristics of the copper metallized SPDT switch exhibited an insertion loss of $0.33 \mathrm{~dB}$ and an isolation of $36.7 \mathrm{~dB}$ at $2.5 \mathrm{GHz}$, the performance is comparable to the SPDT switches fabricated using conventional Au interconnects. High power handling capability was achieved with $\mathrm{P} 1 \mathrm{~dB}$ of $27 \mathrm{dBm}$

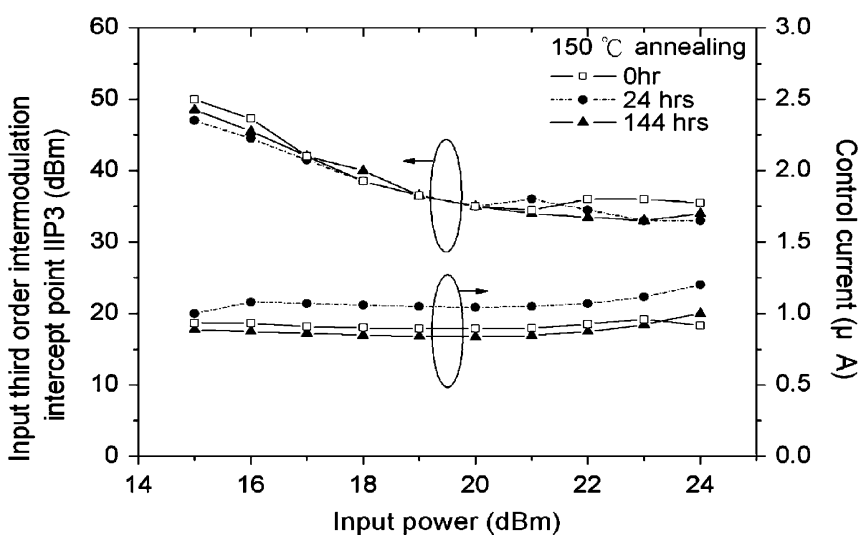

Fig. 6. Input IP3 and control current of the copper metallized SPDT switches with different input power levels at $2.5 \mathrm{GHz}$ after annealing at $150{ }^{\circ} \mathrm{C}$ for different annealing periods.

and IIP3 of $50 \mathrm{dBm}$. Based on the high temperature reliability tests including thermal stress test (annealing at $250^{\circ}$ for $20 \mathrm{~h}$ ) and HTSL test, no significant changes in the dc and RF characteristics were observed for the SPDT switches after these tests. It is evident from these data that the copper metallization process developed is very reliable and can be used for the GaAs MMICs fabrication.

\section{ACKNOWLEDGMENT}

The authors would like to acknowledge Dr. S. H. Tsai and H. C. Huang for experimental assistance.

\section{REFERENCES}

[1] K. Holloway and P. M. Fryer, "Tantalum as diffusion barrier between copper and silicon," Appl. Phys. Lett., vol. 57, no. 17, pp. 1736-1738, Oct. 1990.

[2] K. Holloway, P. M. Fryer, C. Cabral, Jr., J. M. E. Harper, P. J. Bailey, and K. H. Kelleher, "Tantalum as a diffusion barrier between copper and silicon: Failure mechanism and effect of nitrogen additions," $J$. Appl. Phys., vol. 71, no. 11, pp. 5433-5444, 1992.

[3] D. S. Yoon, H. K. Baik, and S. M. Lee, "Effect on thermal stability of a $\mathrm{Cu}$-Ta-Si heterostructure of the incorporation of cerium oxide into the Ta barrier," J. Appl. Phys., vol. 83, no. 12, pp. 8074-8076, 1998.

[4] C. Y. Chen, E. Y. Chang, L. Chang, and S. H. Chen, "Backside copper metallisation of GaAs MESFETs using TaN as the diffusion barrier," IEEE Trans. Electron Devices, vol. 48, no. 6, pp. 1033-1036, Jun. 2001.

[5] H. C. Chang, E. Y. Chang, Y. C. Lien, L. H. Chu, S. W. Chang, R. C. Huang, and H. M. Lee, "Use of WNx as diffusion barrier for copper airbridged low noise GaAs PHEMT," Electron. Lett., vol. 39, no. 24, p. $1763,2003$.

[6] S.-W. Chang, E. Y. Chang, D. Biswas, C.-S. Lee, K.-S. Chen, C.-W. Tseng, T.-L. Hsieh, and W.-C. Wu, "Gold-free fully Cu-metallized InGaP/GaAs heterojunction bipolar transistor," Jpn. J. Appl. Phys., vol. 44 , no. 1 A, pp. 8-11, 2005.

[7] F. Gao, "Reliability evaluation on dual-etch-stop InGaAs PHEMTs," JEDEC, pp. 95-97, 2002.

[8] C.-K. Lin, W.-K. Wang, Y.-J. Chan, and H.-K. Chiou, "BCBbridged distributed wideband SPST switch using $0.25-\mu \mathrm{m}$

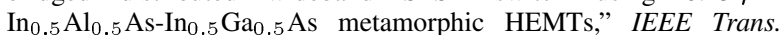
Electron Devices, vol. 52, no. 1, pp. 1-5, Jan. 2005.

[9] H. Ishida, Y. Hirose, T. Murata, Y. Ikeda, T. Matsuno, K. Inoue, Y. Uemoto, T. Tanaka, T. Egawa, and D. Ueda, "A high-power RF switch IC using AlGaN/GaN HFETs with single-stage configuration," IEEE Trans. Electron Devices, vol. 52, no. 8, pp. 1893-1899, Aug. 2005.

[10] K.-H. Lee, Z. Jin, and K.-H. Koo, "High linearity SPDT switch for dual band wireless LAN applications," in Proc. IEEE APMC'05 Conf., 2005, [CD ROM]. 